\title{
Transient Anal Sphincter Relaxations Are a Normal Phenomenon in Healthy Subjects: Author's Reply
}

TO THE EDITOR: We appreciate the opportunity to respond to the letter by Huizinga et al $^{1}$ about our study. The letter addresses the following questions (Figure): (1) Is spontaneous anal relaxation (SAR) a typical feature of fecal incontinence (FI) and a major finding?, (2) Is SAR an appropriate term?, and (3) Does the assessment of SAR lead to unnecessary concern for patients?

Although our study showed that SAR was more commonly found in patients with FI, we acknowledge that, as Huizinga pointed out, SAR is not a representative characteristic of FI. Previous studies have reported that $\mathrm{SAR}$, unstable internal anal sphincter tonic contraction, likely plays a major role in the development of $\mathrm{FI}^{2-4}$ It should be acknowledged, however, that SAR may develop in patients with $\mathrm{FI}$ and constipation. In our study, the proportion of constipation among SAR patients was $47.8 \% .^{5}$ Given that, SAR is unlikely to contribute to FI.

A recent healthy volunteer study using high-resolution colonic manometry (HRCM) indicated that SAR without any motor patterns such as high-amplitude propagating pressure waves or simultaneous pressure wave were found in 10 of 19 subjects. ${ }^{6}$ Their HRCM study lasted 6-8 hours per person. The total number of SAR episodes was 24 in the study, and thus it can be estimated that about 0.1-0.2 episodes per hour are observed in healthy subjects. Given the stabilization and rest period time, ranging from 5 minutes to 10 minutes during HR-AMR study, we believe that SAR during high resolution-anorectal manometry (HR-ARM) examination very rarely occurs in healthy volunteers. In other words, SAR is not

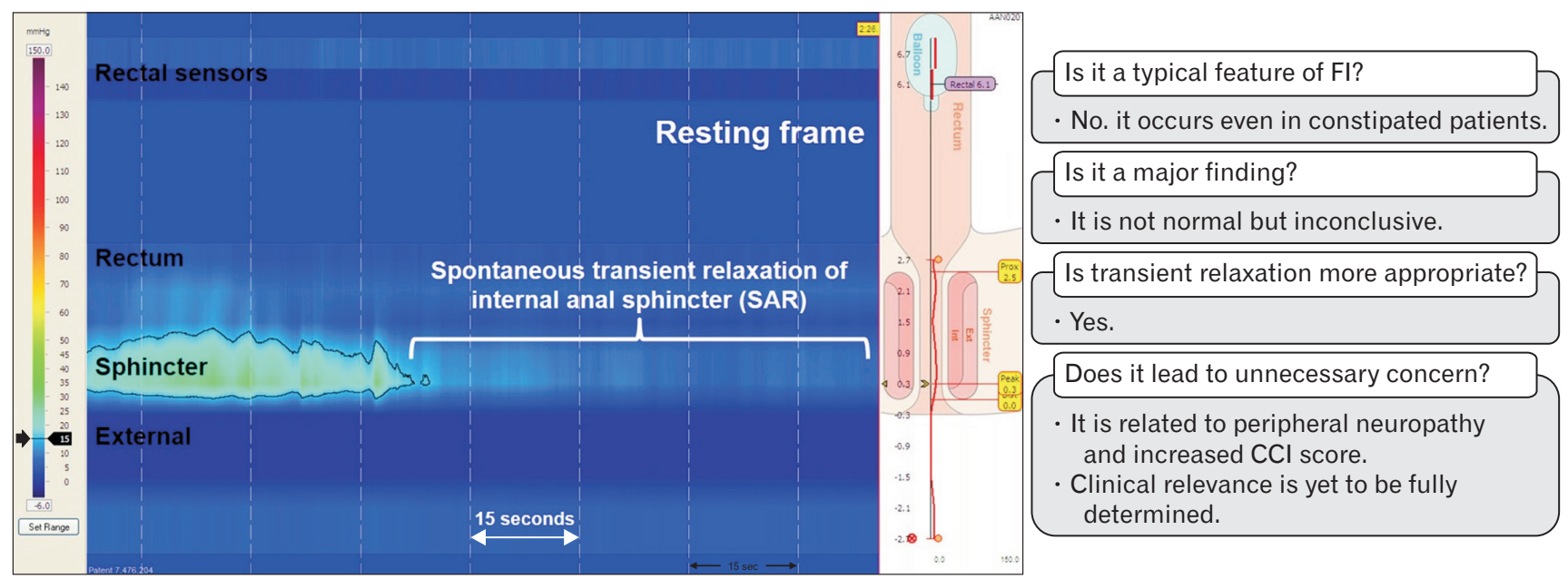

Figure. Main questions and our responses regarding spontaneous transient relaxation of the internal anal sphincter (SAR). FI, fecal incontinence; CCI, Charlson comorbidity index. 
a major finding that is likely to represent a physiological alteration associated with symptom generation, but this is an inconclusive pattern that is seen in patients with anorectal symptoms. Abnormal ARM patterns such as poor propulsion or dyssynergia can be seen in healthy volunteers, but these abnormal patterns are not considered as a normal finding. We acknowledge that SAR is rarely seen in control subjects and may represent a physiological alteration associated with anorectal symptoms, though the relevance is yet to be fully determined.

Unlike Huizinga et $\mathrm{al}^{1}$ who mentioned that anal relaxation is always related to colonic motor events, SAR also occurs without colonic motor events. ${ }^{6}$ HR-ARM examination cannot accurately determine that anal relaxation develops without any colonic motor events. However, we checked gas expulsion and liquid/solid outflow during HR-ARM examinations. We ruled out anal relaxation associated with gas expulsion or liquid/solid outflow in the determination of SAR. Even so, as Huizinga et $\mathrm{al}^{1}$ pointed out, we agree that transient anal relaxation (TAR) is a more appropriate term in HRARM examinations.

TAR may be an inconclusive finding and thus, we do not strongly recommend the evaluation of TAR during HR-ARM examination to assess morbidity and mortality risks in patients with anorectal disorders. In our study, TAR patients (median score $=$ 5) had a higher median Charlson comorbidity index score than controls (median score $=4, P=0.003$ ). ${ }^{5}$ Peripheral neuropathy was also significantly more common in TAR patients $(39.1 \%)$ than in controls $(11.6 \%, P=0.022) .{ }^{4}$ There was a significant difference between the TAR patients (72.7\%) and the controls (41.2\%) regarding the presence of diseases that potentially cause peripheral neuropathy. ${ }^{6}$ Additionally, the TAR patients had a significantly higher incidence of diseases that potentially cause peripheral neuropathy. ${ }^{5}$ There is, meanwhile, a controversy that FI and constipation is related to increased mortality. ${ }^{7,8}$ This controversy might be due to the presence of the accompanying TAR finding. Further research focused on in-depth analysis of TAR are thus urgently needed and enable us to answer the question that TAR is a clinically important finding in patients with defecatory disorders.

Tae Hee Lee

Institute for Digestive Research, Digestive Disease Center, Soonchunhyang University Seoul Hospital, Seoul, Korea

1. Huizinga JD, Milkova N, Chen JH. Transient anal sphincter relaxations are a normal phenomenon in healthy subjects. J Neurogastroenterol Motil 2020;26:552-553.

2. Farouk R, Duthie GS, Pryde A, McGregor AB, Bartolo DC. Internal anal sphincter dysfunction in neurogenic faecal incontinence. Br J Surg 1993;80:259-261.

3. Farouk R, Duthie GS, MacGregor AB, Bartolo DC. Evidence of electromechanical dissociation of the internal anal sphincter in idiopathic fecal incontinence. Dis Colon Rectum 1994;37:595-601.

4. Sun WM, Katsinelos P, Horowitz M, Read NW. Disturbances in anorectal function in patients with diabetes mellitus and faecal incontinence. Eur J Gastroenterol Hepatol 1996;8:1007-1012.

5. Lee TH, Lee JS, Kim J, et al. Spontaneous internal anal sphincter relaxation during high-resolution anorectal manometry is associated with peripheral neuropathy and higher Charlson comorbidity scores in patients with defecatory disorders. J Neurogastroenterol Motil 2020;26:362-369.

6. Milkova N, Parsons SP, Ratcliffe E, Huizinga JD, Chen JH. On the nature of high-amplitude propagating pressure waves in the human colon. Am J Physiol Gastrointest Liver Physiol 2020;318:G646-G660.

7. AlAmeel T, Andrew MK, MacKnight C. The association of fecal incontinence with institutionalization and mortality in older adults. Am J Gastroenterol 2010;105:1830-1834.

8. Koloski NA, Jones M, Wai R, Gill RS, Byles J, Talley NJ. Impact of persistent constipation on health-related quality of life and mortality in older community-dwelling women. Am J Gastroenterol 2013;108:11521158.

Financial support: This work was supported by the Soonchunhyang University Research Fund.

Conflicts of interest: None. 